\title{
Trois disques de musique des Aborigènes de
} Taiwan

Enregistrements : Wu Rong-Shun

Ya-Li Gao

\section{(2) OpenEdition}

\section{Journals}

Édition électronique

URL : http://journals.openedition.org/ethnomusicologie/1475

ISSN : 2235-7688

Éditeur

ADEM - Ateliers d'ethnomusicologie

Édition imprimée

Date de publication : 31 décembre 1994

Pagination : 293-296

ISBN : 2-8257-0503-9

ISSN : $1662-372 X$

\section{Référence électronique}

Ya-Li Gao, "Trois disques de musique des Aborigènes de Taiwan », Cahiers d'ethnomusicologie [En ligne], 7 | 1994, mis en ligne le 03 janvier 2012, consulté le 21 avril 2019. URL : http:// journals.openedition.org/ethnomusicologie/1475

Ce document a été généré automatiquement le 21 avril 2019

Tous droits réservés 


\title{
Trois disques de musique des Aborigènes de Taiwan
}

\author{
Enregistrements : Wu Rong-Shun
}

Ya-Li Gao

\section{RÉFÉRENCE}

The Songs of the Bunun Tribe. Enregistré entre 1987 et 1989. Wind Record (Taipei) TCD-1501

[The Music of the Aborigines on Taiwan Island, vol. 1]

Polyphonic Music of the Ami Tribe. Enregistré le 15.07 et le 13.10.1992. Wind Record (Taipei) TCD-1502 [The Music of the Aborigines on Taiwan Island, vol. 2]

The Songs of the Yami Tribe. Enregistré entre 1992 et 1993. Wind Record (Taipei) TCD-1503

[The Music of the Aborigines on Taiwan Island, vol. 3].

1 A l'aube du XXe siècle, début de l'occupation de Taiwan par les Japonais, des fonctionnaires nippons avaient commencé des enquêtes sur la musique des aborigènes de Taïwan (sauf celle de l'ethnie Pinpu). Le résultat avait été publié en 1922 sous le titre Rapports des enquêtes sur les Aborigènes de Taïwan (8 volumes). Plus tard, des musicologues japonais comme Tanabe, Kurosawa, Takatomo et le musicologue taiwanais Chang $\mathrm{Fu}-$ Hsing avaient participé à ces recherches. Ainsi, les premiers enregistrements avaient-ils été publiés : un disque de Tanabe, enregistré en 1922, réédité en 1978 (Toshiba TW-80011) et deux disques de Kurosawa enregistrés en 1943, édités en 1974 (Sony SJL-78-9M). Une partie de ces enregistrements a été rééditée, d'abord en disque $78 \mathrm{t}$. dans la Collection universelle de musique populaire enregistrée par Constantin Brăiloiu entre 1951 et 1958, ensuite sous forme de $33 \mathrm{t}$. par les Archives internationales de musique populaire (VDE 30-462).

2 Pendant les années soixante, les musicologues taiwanais avaient commencé à recueillir systématiquement la musique des aborigènes. Parmi ces chercheurs, seuls Lu Ping-Chuan et Hsu Tsang-Houei ont laissé des archives précieuses : pour Lu, trois disques édités en 1977 chez Sony, réédités en 1980 par The Chinese Folk Arts Foundation; pour Hsu, trois 
disques édités en 1979 par cette même fondation, et un autre : Chants de travail et d'amour des peuples aborigènes de Taïwan, paru en 1985 chez Arion (ARN 33785).

3 Progressivement, de jeunes chercheurs comme Chu Yang-Liang, Lin Hsin-Lai, Li CheYang, Ming Li-Guo, Wu Rong-Shung et Chen Shan-Hua, se sont joints à cette enquête et ont recueilli bon nombre d'enregistrements inédits. Or, la conversion chrétienne des aborigènes et, à un moindre degré, leur changement de mode de vie, ont été si importants que leurs traditions et leurs musiques sont en voie d'extinction : aujourd'hui, il est très difficile pour les chercheurs de trouver autant de musiques et, à Taiwan, en dehors de la parution des archives de $\mathrm{Lu}$ et $\mathrm{Hsu}$, il n'y avait pratiquement aucune publication d'enregistrements sur la musique aborigène jusqu'à l'édition de la série The Music of the Aborigines on Taiwan Island de Wind Record.

4 En Europe, grâce à la Collection universelle de musique populaire enregistrée du Musée d'ethnographie de Genève, les musicologues occidentaux ont découvert la musique aborigène de Taiwan et ont commencé à s'y intéresser. Récemment, trois CD ont paru sur cette musique : en 1989, Musique des peuples minoritaires de Taiwan, enregistré par Chen Shui-Cheng, édité chez Arion (ARN 64109) et Polyphonies vocales des Aborigènes de Taiwan, enregistré en France, à la Maison des Cultures du Monde, à l'occasion des concerts donnés par les Aborigènes de Taiwan ${ }^{1}$, paru chez Inédit (W 260011); en 1991, Taiwan - Music of the Aboriginal Tribes, enregistré par Wolfgang Laade ${ }^{2}$, édité chez Jecklin-Disco (JD 653-2) dans la série « Music of Man Archive ».

$5 \quad$ Les trois CD édités par Wind Record concernent trois groupes ethniques: les Bunun, les Ami et les Yami. Ils nous permettent de comprendre d'autant mieux leurs musiques et leurs cultures que la réalisation en est ambitieuse et très soignée : d'abord, la qualité du son est remarquable ; ensuite, les notices comportent des transcriptions phonétiques des textes vernaculaires, traduits et commentés en chinois et en anglais; enfin, quelques photos et des transcriptions musicales sur portée illustrent le livret d'accompagnement.

6 L'ethnie Bunun est un peuple qui valorise tout à la fois la compétence individuelle dans les activités collectives et la valeur du groupe devant le public. Ses procédés de chant sont toujours homorythmiques et polyphoniques : plusieurs voix chantent simultanément et progressent horizontalement sur des accords consonants. Cette manière de chanter est propre aux Bunun. Leur Pasi but but, en particulier, a attiré l'attention des musicologues du monde entier ${ }^{3}$ : il s'agit d'un chant de prière, chanté en chromatisme ascendant, destiné à apporter une abondante récolte de millet. Ce chromatisme progresse par différents mouvements et par imitation à différentes hauteurs, chaque voix se caractérisant par une certaine indépendance.

7 Le répertoire du disque The Songs of the Bunun Tribe peut se diviser en deux parties: les chants et les solos instrumentaux. Selon les circonstances dans lesquelles ils sont exécutés, on peut distinguer cinq catégories : chants de rituels, chants d'enfants, chants de divertissement, chants de travail et ballades.

8 Les chants rituels et enfantins sont les plus nombreux. Curieusement, chez les Bunun, nous ne trouvons ni chants d'amour, ni danses. Quant aux instruments de musique, ils sont peu nombreux : l'arc musical, la guimbarde, le pilon en bois. En fait, les Bunun en jouent pour se divertir.

9 Si nous confrontons les répertoires des archives enregistrées par Lu et Hsu et celui de Wu, nous nous rendons compte que la sélection faite par Wu est plus riche. Pour chaque 
catégorie de musique, il y a plus de variété. En particulier, nous trouvons des refrains ${ }^{4}$ dont les paroles sont très intéressantes dans le chants d'enfants de la plage 6 .

Parmi tous les aborigènes de Taiwan, les Ami sont les plus nombreux. Ce peuple est connu pour sa société matrilinéaire et sa stricte hiérarchie sociale. Dans un groupe, les chanteurs appartiennent souvent à une même strate sociale et ont à peu près le même âge. Ils participent toujours ensemble à toutes les activités quotidiennes. Ainsi, qu'ils chantent ou qu'ils travaillent, ils s'accordent parfaitement. Leurs chants sont principalement polyphoniques. Le procédé le plus particulier est celui des Beinan, un sous-groupe Ami, qui utilise le contrepoint, souvent à deux, trois ou quatre voix. Par ailleurs, leurs chants demandent une grande capacité d'improvisation et toutes les voix doivent finir sur une même note. Souvent, leurs chants s'associent à la danse et se lient aux rituels : ceux des fêtes calendaires, du cycle de la vie. Le rituel le plus important est celui qui marque une récolte abondante: ses mélodies sont souvent chantées en style responsorial.

Le répertoire du disque Polyphonic Music of the Ami Tribe a été enregistré chez les Beinan. Ces documents comprennent trois catégories correspondant à des circonstances précises : chants de rituels, chants de travail et chants de divertissement.

La confrontation entre les archives de $\mathrm{Hsu}$ et $\mathrm{Lu}$ et le répertoire publié par Wu fait apparaitre que le nombre des chants pour fêter une récolte abondante s'est réduit considérablement et que les chants d'amour et les chants d'enfants sont absents. C'est bien regrettable! D'autant plus qu'aujourd'hui, le rituel pour fêter une récolte abondante est devenu un grand festival destiné à attirer les touristes et qu'à cause de cette tendance, leurs musiques et leurs danses se modifient. D'autre part, nous ne savons pas combien de chants traditionnels existent encore dans ce rituel. En revanche, sur ce disque, bon nombre de chants de travail ont été improvisés par les chanteurs. Leur capacité d'improvisation est tellement développée qu'il composent facilement leur chant sur des sujets très variés.

13 Les Yami sont le seul peuple qui habite dans la petite île de Lanyu (aussi nommée Botel Tobago), d'une superficie de $45 \mathrm{~km}^{2}$, située au sud-est de Taiwan. Leur mode de vie, étroitement lié à la pêche, et l'importance qu'ils accordent à la construction et à l'inauguration des maisons ont beaucoup influencé leur musique. En outre, leurs danses sont très célèbres parmi les aborigènes. Généralement, elles comprennent deux catégories: celles liées à un rituel (par exemple, l'inauguration) et celles pour le divertissement. Ces dernières sont souvent dansées seulement par les femmes. Par rapport aux chants des Bunun et des Ami, il est évident que ceux des Yami sont fort « simples » : leur procédé est principalement l'homophonie récitative et les «mélodies » sont souvent composées de deux, trois ou quatre notes dont les intervalles fluctuent entre les demi-tons et les tierces. De plus, on peut facilement percevoir de l'hétérophonie quand ils chantent en chœur.

14 Sur le disque The Songs of the Yami Tribe, les enregistrements sont très nombreux et les répertoires sont assez variés : chants consacrés à l'inauguration des maisons, à la pêche, à l'agriculture, à l'amour; chants liés à la danse, berceuses, chant de divertissement et ballade. Curieusement, ces chants sont extrêmement brefs (ils durent de 0'10" à 4'18"; plus de la moitié durent moins de 1'30", y compris deux chants de 0'44" et 0'24" liés à la danse). 

souvent chantées avec des paroles différentes, par des personnes différentes, en des occasions différentes. On peut se demander s'il s'agit de leur tradition et s'il existe des significations attachées à ces mélodies souvent utilisées. Dans ce $\mathrm{CD}$, on trouve également deux chants très "mélodiques" (plages 20 et 22) dont le style semble influencé par une culture tout à fait différente. Il est étonnant qu'ils aient été adoptés et qu'ils soient considérés comme des chants yami. Actuellement, ces chants sont enregistrés hors contexte, d'une part, parce que ces aborigènes ont beaucoup changé de mode de vie et qu'ils ne pratiquent plus certains chants; d'autre part, parce que les gens capables de chanter des airs traditionnels sont peu nombreux. Il faut donc faire preuve de circonspection dans l'étude et l'interprétation de ces différents " patrimoines ».

Bien que ces deux derniers disques n'apportent que peu d'éléments nouveaux par rapport aux disques antérieurs, il faut néanmoins féliciter Wu pour la qualité technique des enregistrements effectués sur le terrain et le soin apporté à la réalisation des livrets qui seront désormais indispensables à tout chercheur intéressé par la musique des aborigènes de Taiwan.

\section{NOTES}

1. En 1988, les chanteurs aborigènes de Taïwan (principalement les Ami, Bunun, Paiwan et Rukai) ont été invités par la Maison des Cultures du Monde à donner des concerts à Paris. Certains enregistrements publiés à cette occasion ont été complétés par des archives recueillies pendant les années soixante à Taïwan.

2. Deux chercheurs taïwanais, Wu Rong-Shun - l'auteur des trois disques dont il est question ici et Lin Qing-Cai, ont assisté Laade dans son enquête sur le terrain.

3. Ce chant a été édité dans la Collection universelle de musique populaire enregistrée : disque $78 \mathrm{t}$. $\mathrm{N}$ - 21 , face 1a, sous le titre « Chant rituel pour les semailles du millet ».

4. Dans ce chant, chaque vers compte cinq mots, et les deux ou trois derniers mots d'un vers sont les premiers du vers suivant. Par exemple, $1^{\mathrm{er}}$ vers : $\mathrm{ABCDE}, 2^{\mathrm{e}}$ vers : DEFGH, $3^{\mathrm{e}}$ vers : FGHIJ, $4^{\mathrm{e}}$ vers : IJKLM, et ce jusqu'à la fin du chant. 